Portland State University

PDXScholar

\title{
Tell Us More: Reading Comprehension, Engagement, and Conceptual Press Discourse
}

Dot McElhone

Portland State University

Follow this and additional works at: https://pdxscholar.library.pdx.edu/edu_fac

Part of the Curriculum and Instruction Commons, and the Educational Methods Commons Let us know how access to this document benefits you.

\section{Citation Details}

McElhone, Dot, "Tell us more: reading comprehension, engagement, and conceptual press discourse" (2012). Education Faculty Publications and Presentations. Paper 11. http://archives.pdx.edu/ds/psu/ 9047

This Post-Print is brought to you for free and open access. It has been accepted for inclusion in Education Faculty Publications and Presentations by an authorized administrator of PDXScholar. Please contact us if we can make this document more accessible: pdxscholar@pdx.edu. 


\title{
Running Head: CONCEPTUAL PRESS DISCOURSE
}

Tell Us More: Reading Comprehension, Engagement, and Conceptual Press Discourse

\author{
Dot McElhone
}

Portland State University 


\begin{abstract}
This study examines interactions between teachers and students during reading comprehension instruction to determine how certain patterns of teacher-student talk support student comprehension achievement and reading engagement. The central focus of the study is conceptual press discourse, a pattern of teacher response that includes requests for evidence, examples, clarification, and elaboration. Hierarchical Linear Modeling analysis of data from 21 fourth- and fifth-grade classrooms (495 students) indicated that in classrooms where teachers more frequently used discourse patterns that reduced conceptual press, students demonstrated weaker comprehension and engagement outcomes.
\end{abstract}


Tell Us More: Reading Comprehension, Engagement, and Conceptual Press Discourse A cluster of fourth graders is gathered on the carpet of their classroom as their teacher reads aloud to them the final pages of Thank You, Mr. Falker by Patricia Polacco. She closes the book, pauses, and asks, "How do you think Trisha changed during the story?” Several hands shoot up in the air. The teacher looks over her class, then calls on Ana.

"She got to be happier," Ana suggests.

This is the moment when my research begins. What happens next? And does it matter? We might expect the teacher to paraphrase Ana’s response ("So you think she got happier”), evaluate it ("Good"), or even record it on a class chart, and then call on another student. Each student would contribute his or her idea, and the teacher would respond to each in turn. She might not evaluate every student's response in the traditional initiation-response-evaluation (IRE) pattern of talk (Mehan, 1979; Cazden, 1988), but the norm would likely be one contribution per student, and on to the next child.

The central focus of the study is conceptual press discourse, a pattern of teacher-student talk that challenges students to think beyond their initial responses in the analysis of texts and in the use of comprehension strategies. This study explores what happens if the teacher turns the conversation back to Ana. What if the teacher asks Ana, "Where can you find evidence for that in the text?" And when Ana answers this question, what if the teacher asks her, "How does that help you understand the story?” In short, what if the teacher presses Ana to keep thinking? And what if Ana and her classmates aren’t surprised by their teacher's follow up questions because this is the normal pattern of teacher-student talk in their classroom? What if the students are accustomed to having their initial responses challenged, extended, pressed? Will they grow as 
readers? Will they become more motivated to think strategically as they read? Might they even find reading more interesting and enjoyable?

In recent years, researchers and practitioners have paid increasing attention to the role of classroom talk in supporting students' engagement with reading and their abilities to actively and strategically construct meaning from texts. This emphasis on classroom discourse is based on an understanding of discussion as a powerful social context for learning. Vygotsky viewed learning as occurring on two planes: the social or interpersonal and the individual or intrapersonal (1978). Discussions can enable students to participate socially in strategic thinking and reasoning about texts and to observe their peers and teacher's uses of these important tools for effective comprehension. These tools can then be internalized or appropriated into students' thinking about texts as they read independently. "From a sociocultural perspective, discussion enables students to co-construct knowledge and understandings about the text and internalize the ways of thinking that foster the knowledge, skills, and dispositions needed to transfer to the reading of new texts (Wells, 2007)” (Lawrence \& Snow, 2011, p. 369).

Researchers have also found links between discussion and engagement (cf. Almasi, McKeown, \& Beck, 1996). Nystrand and Gamoran drew a distinction between procedural engagement, which “reflects an accommodation to classroom rules and regulations” and substantive engagement, which "involves a sustained personal commitment to understanding the world of a story or poem, as well as literary and other issues raised by the work itself” (1991, p. 262). These researchers found that teacher-student discourse patterns shaped the character of student engagement along a continuum of disengagement, procedural engagement, and substantive engagement. When teachers worked “students' answers into the fabric of an unfolding exchange, ... [built] on the substance of what the student [said], ... and [followed up] 
on student answers by incorporating these answers into subsequent questions,” (p. 264), students were more substantively engaged.

Reading motivation and engagement are valuable in their own right and are also positively associated with achievement-related outcomes, such as standardized tests and grades (Connell, Spencer, \& Aber, 1994; Skinner, Wellborn, \& Connell, 1990). In Nystrand and Gamoran’s words, “significant academic achievement is not possible without sustained, substantive engagement” (1991, p. 262). Reading engagement is so strongly linked to achievement that "engagement in reading may substantially compensate [on measures of reading achievement] for low family income and educational background” (Guthrie \& Wigfield, 2000, p. 404). It is essential to develop and refine approaches to classroom discourse that support both engagement and achievement in reading.

Scholars have suggested and researched multiple approaches to literacy instruction that emphasize opportunities for students to engage in discussion, such as Questioning the Author (cf. Beck \& McKeown, 2006), Book Clubs (cf. Raphael \& McMahon, 1994), and Junior Great Books Shared Inquiry (cf. Solomon, 1990). Such approaches “are characterized by some type of instructional frame that describes the moves of the teacher, the role of the text, specific metacognitive strategies, and benchmarks of success” (Murphy, Wilkinson, Soter, Hennessey, \& Alexander, 2009, p. 742). In short, these approaches are multifaceted. In their review of oral discourse and comprehension, Lawrence and Snow respond to this complexity by suggesting that future research ask, "Precisely what features of classroom discussion account for its positive effects on student learning?” (2011, p. 332).

Further, while multifaceted programs have in many cases yielded positive results (Murphy, et al., 2009), one challenge they face is implementation. Historically, multifaceted, 
complex approaches to comprehension instruction (e.g., transactional strategies instruction, Pressley, et al., 1992) have proven difficult for teachers to learn to implement well and sustainably (Wilkinson \& Son, 2011, p.366). Murphy and her colleagues found that the vast majority of studies on approaches to classroom discussions around texts were "were conducted by researchers who played a primary role in the creation of a given approach” and explained that "it is not clear whether other researchers or teachers could replicate the effects reported by the originators” (2009, p.752). Beyond the challenge to teachers of learning new programs, the current policy climate around reading instruction also limits the likelihood of teachers implementing larger scale instructional approaches beyond those mandated at the school or district level (such as core reading programs). These circumstances call for research into smaller scale, more flexible approaches to strengthening classroom discourse with the potential to be more easily learned and implemented by teachers. Despite myriad constraints on their instructional practices, most teachers still have freedom to act on their professional judgment in the context of the moment-by-moment interactions they have with students.

\section{Current Study}

This study examines a pattern of teacher-student talk called conceptual press discourse, which involves responding to student contributions by pressing them to take their thoughts further. I developed conceptual press discourse to meet the need in the field for a small-scale, adaptable way to approach classroom talk and to better understand how particular features of classroom talk might account for positive effects on students’ reading comprehension and engagement.

Previous research has consistently found that the teacher-dominated Initiate-RespondEvaluate (IRE) pattern of interaction, in which a teacher poses a question, a student responds, 
and the teacher evaluates that response, is ubiquitous in instruction (cf. Mehan, 1979; Cazden, 1988). Nystrand characterizes this pattern of “recitation” as inherently monologic and complains that "by evaluating student answers rather than responding to student comments and ideas, teachers effectively thwart dialogue” (1997, p.12).

Cazden (1988) frames reading comprehension as an "active construction by each student of 'contexts in the mind'” and claims that teachers are in a position to help students through this process "in the molar form of the entire curriculum, [and] in the molecular form of what is said in the E slot" (Cazden, p. 116). Nystrand also emphasizes the importance of the "E slot" in teacher-student discourse.

In short, how students think-indeed the extent to which they really need to think in school-and consequently what they can learn depend a lot on how their teachers respond to their students' responses. This is the most fundamental way that classroom discourse shapes student learning (1997, p. 29).

Nassaji and Wells suggest that when a student offers a non-standard interpretation or response, “instead of negatively evaluating [that] student's response or providing the required information in a comment, the teacher can equally ask a further question to the previous speaker, or any other students, in order to obtain a more adequate answer” (Nassaji \& Wells, 2000, p. 379). Whether or not we accept obtaining an "adequate answer” as the purpose of teacherstudent interaction, Nassaji and Wells point to the range of options teachers have when responding to student responses. These researchers found that "it was the choice of follow-up move that largely determined how the discourse developed” (2000, p. 382). Students in this study were more likely to offer longer, more complex contributions to discussions during sequences in which teachers used less evaluative follow-ups. 
Similarly, Boyd and Rubin found that "the distinguishing characteristic of teacher questions that elicited extended student talk was found to be their contingency on previous student utterances rather than whether they were open-ended or inquired about known information” (2006, p. 141). This finding aligns with Nystrand and Gamoran’s (1991) report of the important role of uptake in facilitating student engagement in classroom discourse (and subsequent learning). Nystrand defined uptake as the "incorporation of previous answers into subsequent questions” (1997, p. 36) and described discourse characterized by frequent teacher uptake of student ideas as more dialogic than IRE discourse. These studies demonstrate that teacher responses to student utterances shape the character of discourse in classrooms and have the potential to support or undermine student thinking and learning. They suggest that teachers should aim to "take up" students' ideas in a non-evaluative manner when they respond to student utterances.

According to cognitive evaluation theory (a component of self-determination theory), two essential components of intrinsically motivating environments are optimal challenge and autonomy support (Deci \& Ryan, 1985). Teachers offer learners optimal challenges when they provide tasks that push them toward, but not past, their cognitive limits. Deci and Ryan found that in the face of such challenges, "people work to conquer them, and do so persistently" (p.33). Instruction that supports autonomy provides students with opportunities to do and think for themselves. Such approaches have been highlighted as essential to promoting both general engagement and engaged reading (Fredricks, Blumenfeld, \& Paris, 2004, p.75; Guthrie \& Wigfield, 2000; Baker, Dreher, \& Guthrie, 2000).

High conceptual press discourse moves include responding to student contributions by asking for clarification, elaboration, evidence, or examples. Kachur and Prendergrast 
characterized a "request for elaboration not [as] an attempt to push the student toward the 'right' answer, but an attempt to encourage the student to explore her own 'interpretive horizons'” (1997, p.83). A high-press response to a student idea, such as a request for evidence, can both encourage a student to examine his own thinking and push him to re-engage with the text itself. A sociocultural perspective suggests that as students are challenged to elaborate, clarify, or back up their comments with textual evidence during teacher-student dialogue, they will begin to internalize these discourse processes into inner speech about and during independent reading. If students engage in more elaborated, precise, and evidence-based thinking during reading, they should more effectively construct meaning from texts and demonstrate stronger comprehension achievement. By following up on the ideas students present with optimally challenging responses, teachers can allow students to drive classroom discussions and to experience autonomy as thinkers. In this way, conceptual press discourse moves have the potential to support not only students' reading comprehension, but also their intrinsic motivation to read and their engagement with the process of reading. Blumenfeld, Puro, \& Mergendoller (1992) found that when teachers assigned challenging tasks and "pressed for understanding” (Fredricks, Blumenfeld, \& Paris, 2004, p.75), middle school students rated their cognitive engagement and use of metacognitive strategies higher.

By pressing students to take what Rosenblatt (1978/1994) termed a more efferent stance, characterized by an emphasis on the information a reader can take away from a text, rather than on the feelings and attitudes brought about through reading, teachers can "foster student talk and high level comprehension of literature (Soter \& Rudge, 2005)” (Boyd \& Rubin, 2000, p.143). Conceptual press discourse moves may allow teachers to move children beyond their initial, often aesthetic-expressive responses to texts and push them to both dig deeper into the content of 
texts (efferent stance) and reach higher in their critical thinking about texts (critical-analytic stance).

In contrast to high conceptual press moves, such as requesting examples, French and MacLure (1981) describe reformulators, which progressively decrease the cognitive load for the child (Cazden, 1988, p. 109). Examples of these discourse moves, which reduce the level of conceptual press on students, include narrowing initially open-ended questions by offering a limited number of answer choices or providing hints about the correct answer. These pressreducing moves may limit student engagement with discussions and texts, and thereby limit learning.

This study examines interactions between teachers and students during reading comprehension instruction to determine how specific patterns of teacher-student talk support student comprehension achievement and engagement. Engagement is a multi-dimensional construct that includes affective, behavioral, and cognitive components. The relationship between motivation and engagement is complex. Behavioral engagement, which can be observed as active participation in a task or activity, could be seen as the outcome of intrinsic or extrinsic motivation. Fredricks, Blumenfeld, and Paris (2004) explain that “emotional engagement refers to students' affective reactions in the classroom, including interest, boredom, happiness, sadness, and anxiety (Connell \& Wellborn, 1991; Skinner \& Belmont, 1993)” (p. 63). This study focuses on the emotional or affective engagement related to intrinsic motivation. In this context, affective engagement refers to enjoyment of, positive feelings toward, and sense of competence about an activity: the "I want to" and "I can” feelings associated with intrinsic motivation. Cognitive engagement encompasses thinking about the task at hand, similar to concentration. Under ideal instructional circumstances, students are intrinsically motivated to participate in learning 
activities, experience positive affect that draws them further into the activities, and are actively thinking as they engage in those activities. I examine intrinsic motivation for reading (affective engagement), strategic reading (cognitive engagement), and the choice to read on one's own time (behavioral engagement) as distinct outcomes in order to better understand which aspects of reading engagement may be most easily leveraged through classroom discussion.

Teachers and students in 21 fourth- and fifth-grade classrooms were followed across the course of one school year. Teachers were not trained to use conceptual press discourse, nor were they informed of the study's focus on discourse patterns. Variations across the data set in the degree to which teachers used conceptual press discourse moves were naturally occurring. I used this naturalistic approach rather than an intervention in order to better understand the patterns of talk already occurring in classrooms and because of my concern about ease of future implementation. If I were to find that a pattern of talk some teachers were already using without specific training predicted strong growth in reading comprehension achievement or engagement, such a pattern would arguably be easier to pass on to other teachers than would a multifaceted program originating with researchers.

Patterns of teacher discourse were recorded using a researcher-designed observation protocol over the course of three observations of reading comprehension instruction. Students' engagement was measured using pre- and post-surveys. Murphy and her colleagues concluded their recent meta-analysis of the effects of classroom discussion on reading comprehension calling for "many more [quantitative] multiple-group studies... particularly ones in which commercially available assessments are employed as outcome measures” (2009, p. 761). This study answers that call by measuring reading achievement through a pre- and post-administration of the comprehension portion of the Gates-MacGinitie Reading Test (GMRT; MacGinitie, 
MacGinitie, Maria, Dreyer, \& Hughes, 2000). I used hierarchical linear modeling to analyze relationships between teacher discourse patterns and student outcomes.

This study examines the following questions: Do students in classrooms where teachers incorporate more high conceptual press responses (or fewer reducing press responses) demonstrate stronger:

- comprehension achievement,

- $\quad$ intrinsic motivation to read (affective reading engagement),

- $\quad$ use of strategies during reading (cognitive reading engagement), and

- $\quad$ breadth and frequency of self-selected reading (behavioral reading engagement)?

\section{Method}

\section{Participants}

The sample included 21 teachers in 16 California schools. Initially, teachers were recruited from schools participating in the Noyce Foundation's Every Child a Reader and Writer professional development program and from schools recommended by Ellin Keene (Mosaic of Thought, 1997) to increase the likelihood that the sample would include teachers providing instruction in reading comprehension. Prior research has shown a dearth of comprehension instruction; many teachers assess comprehension but spend very little time teaching students about comprehending texts (Durkin, 1978/1979; Pressley, 2006; Snow, 2002). Teachers who had participated in substantial professional development around reading comprehension were selected to increase the probability of including in the sample some very strong reading comprehension teachers. After exhausting contacts acquired from the Noyce Foundation and from Ellin Keene, recruitment attempts widened to include other schools within the districts that 
were already included in the sample, and finally to other districts in the area based on personal contacts.

Table 1 provides background information on the 21 teachers and the demographics of their schools (Fiscal, Demographic, and Performance Data on California’s K-12 Schools, 2008). The teachers, all but two of whom were female, ranged in classroom experience from 1 to 26 years. The schools varied widely in terms of their populations of students qualifying for Free and Reduced Price Meals (3.0\% to 82.0\%) and students identified as English Language Learners (8.8\% to $67.6 \%)$.

\section{Teacher Level Data}

Observations. Instruction was observed three times in each of the 21 classrooms. Each observation lasted approximately one hour. One of the three observations was videotaped. The observations were pre-scheduled with the teachers to ensure that they would occur during reading comprehension instruction. Teachers were encouraged to continue with their "normal, ongoing reading comprehension instruction” rather than teach special lessons for the observations. The first observation in each classroom occurred in November/December 2007, the second in January/February 2008, and the third in February/March 2008.

Coding Teacher Talk. The independent variables for the study: patterns of talk in comprehension instruction (with an emphasis on the level of conceptual press) were coded using a researcher-designed observation protocol. The observation protocol underwent multiple revisions over the course of several pilot observations during the 2006-2007 school year. Protocol development began with detailed field notes taken during observations of fourth- and fifth-grade reading comprehension instruction. The emphasis of these observations was on discourse patterns. A core list of discourse moves, such as paraphrasing student responses, 
narrowing initial questions, and asking students to provide examples arose out of these observations. Through further observations, this list was refined and supplemented. The final protocol included 29 teacher discourse moves and was detailed enough to capture a nuanced picture of classroom discourse, while still allowing discourse moves to be quantified. The frequency of each of these moves was tallied during observations of instruction. At the end of each observation, the frequency with which the teacher used each discourse move and the ratio of each discourse move to total teacher utterances was calculated.

Each of the three classroom observations in each of the 21 classrooms yielded a complete observation protocol displaying the total number of utterances by the teacher during the session, the frequency with which he or she used each of the 29 discourse moves on the protocol, and the duration of the session. Because the purpose of the protocol was to capture relevant patterns of teacher talk and the relative frequencies of various types of discourse moves, each discourse move in a given session was divided by the total number of utterances in that session. A more typical approach in quantified research on teacher behaviors or teacher talk is to divide the frequency of a behavior by the duration of the observation (cf., Chinn, Anderson, \& Waggoner, 2001). For this study that approach would have yielded results such as "number of paraphrases per minute.” Teachers in this study varied widely in the total number of utterances they used in an hour of instruction. Some teachers flooded the classroom with talk, while others interacted with students very little. It seemed that a given discourse move, for example a hint or a request for a student to elaborate on his ideas, might carry different weight in a "flood of teacher talk" classroom than in a classroom with very little teacher talk. If each of these two teachers made one request for examples in a given hour, the request might carry more weight in the classroom where it was one of only a handful of teacher utterances. Any given discourse move in the "flood 
of talk" classroom would likely be drowned out by other talk. Because the teacher discourse variables were meant to capture a teacher's predominant mode of interaction, the proportion of moves to total utterances was the most appropriate way to represent the data. In order to further reduce the teacher discourse data to a more manageable number of variables, for each of the 29 discourse moves, I calculated the mean of the proportions (uses of this discourse move/total utterances) across the three observations.

Teacher Discourse Patterns. Key discourse moves were then grouped into six superordinate discourse patterns (see Table 2). Not all of the 29 discourse moves were grouped into any of these discourse patterns. The first four discourse patterns, High Press Discourse ${ }^{1}$, Expanded High Press Discourse, Sustained High Press, and Reducing Press Discourse were designed to examine hypotheses regarding the relationship between conceptual press discourse and student outcomes.

The High Press Discourse pattern includes discourse moves that consistently pressed students to think further about their ideas. There were a few discourse moves that seemed to press students to think further, but not in all instances. For example, a teacher occasionally asked a student, "What's one word for that?” In some cases, this question served to press a student to articulate her own idea more precisely. In other cases, the question seemed to serve in a "guess what's in the teacher's head” role. Expanded High Press Discourse includes all the moves in the High Press Discourse pattern along with three moves that pressed students to think further, if not in every case. Clearly, only one of these two patterns could be included in statistical models, but because this was an initial study of conceptual press, it was appropriate to examine multiple operationalizations of high press discourse in the form of these two patterns. In contrast to the 
moves in the High Press Discourse patterns, Reducing Press Discourse moves reduce the cognitive load on a student: they make the required thinking less challenging.

During observations, teacher utterances were tallied with the appropriate discourse move from the observation protocol and were further categorized in terms of whether they were part of a sustained interaction. Teacher utterances occurring during an interaction sustained with an individual child or small group over multiple turns were coded “Sustained Discourse.” When a teacher response was a second, third, or further response to an individual or small group within a given interaction, it was tallied in the Sustained Discourse section of the section for the appropriate discourse move. The Sustained High Press Discourse pattern represents the intersection between Sustained Discourse and High Press Discourse.

There was substantial variation across classrooms in the amount of instruction teachers provided on comprehending texts and using comprehension strategies. Prior research indicates that coordinated strategy instruction can be an important contributor to student comprehension achievement and reading engagement (Pressley, 2006; NICHD, 2000; Mason, 2004). Thus, the variation in the amount of strategy instruction could reasonably be expected to impact student outcomes in the study. In order to examine that variation in the analysis, a Strategy Instruction Discourse pattern was included.

A colleague trained to identify the discourse moves included in the discourse patterns watched videos from two of the observed lessons and tallied discourse moves as described here. Interrater reliability was $100 \%$ on the first video and $77.8 \%$ on the second. Differences were resolved through discussion. Remaining coding was conducted independently.

\section{Student Outcome Measures}


Students completed four outcome measures once in the fall of 2007 and again in the spring of 2008. Both fall and spring administrations were spread over two days. Students completed the reading comprehension achievement measure on the first day. On the second day, which usually occurred within a week of the first, students completed three survey measures addressing three facets of reading engagement: affective, cognitive, and behavioral engagement. All four measures were group-administered to whole classes.

Reading Comprehension Achievement. Comprehension achievement was measured using the comprehension subtest of the Gates-MacGinitie Reading Test, Fourth Edition (GMRT; MacGinitie, MacGinitie, Maria, Dreyer, \& Hughes, 2000). The GMRT is a standardized assessment widely used in reading comprehension research. The Kuder-Richardson Formula 20 (K-R 20) reliability coefficients computed by the test publisher for the test forms used range from 0.92 to 0.93 (MacGinitie, et al., 2002). The publishers report extensive validity evidence.

Affective Reading Engagement. Intrinsic motivation for reading (affective engagement) was measured using an abridged form of the Motivations for Reading Questionnaire (MRQ; Wigfield \& Guthrie, 1997). From the original measure, subscales for reading efficacy, preference for challenge, reading curiosity, importance of reading, reading involvement, and the social aspects of reading were used because they were most closely related to the affective aspects of reading motivation. The MRQ includes 29 items addressing students' motivations for reading. Each item offers the same Likert-type four response choices: “Very Different from Me,” “A Little Different from Me,” “A Little Like Me,” and “A Lot Like Me.” The MRQ is an established measure that has been widely used in research on reading motivation. The validity of the MRQ is suggested by the significant correlations between several of its subscales and measures of amount and breadth of reading (cf., Wigfield \& Guthrie, 1997; Baker \& Wigfield, 1999). 
Wigfield and Guthrie found moderate to strong reliabilities for each of the subscales included in the measure (1997).

Cognitive Reading Engagement. Mokhtari and Reichard’s (2002) Metacognitive Awareness of Reading Strategies Inventory (MARSI) was the measure of cognitive reading engagement (represented by student use of reading strategies). This measure presents students with statements about their use of particular reading strategies. Students respond on a 5-point Likert scale ranging from "Never or almost never true of me” to "Always or almost always true of me.” The MARSI was developed through an iterative theoretically and empirically driven process and then "field tested ... with a large sample of students $(\mathrm{N}=825)$ in Grades 6-12 drawn from 10 urban, suburban, and rural districts in five midwestern states” (Mokhtari \& Reichard, 2002, p. 252). Factor analysis of the results yielded three scales: global reading strategies, problem solving strategies, and support strategies. Cronbach’s alpha reliability for the total sample was 0.89. Mokhtari and Reichard found that students who reported stronger overall reading ability also reported more frequent use of metacognitive reading strategies, suggesting construct validity for the measure.

Behavioral Reading Engagement. The Reading Activity Inventory (RAI; Guthrie, McGough, and Wigfield, 1994; Guthrie, Wigfield, Metsala, \& Cox, 1999) provides a measure of the frequency and breadth of students' reading (behavioral engagement). The RAI is a 10-item survey containing two types of items. Odd numbered items ask students whether they have read in a particular genre by choice (rather than as a school assignment) over the past week. If they have read in that genre, they are asked to write down what they remember of the title, author, and topic. Students who circle “No" score one point. Students who circle "Yes” but offer no further information score two points. Students who circle "Yes" and record the topic but no other 
information score three points. Students who circle "Yes" and record the title or author score four points. Genres include fiction, books about sports, books about science, comic books and magazines, and a section for all other genres. Even numbered items ask students how frequently they read in a given genre by choice (regardless of whether they did so in the past week). Responses on the four-point scale range from “Almost never” to “Almost every day.”

The RAI has been modified for use in several studies. For example, Perencevich (2004) used a version of the RAI keyed specifically to reading in science and Wigfield and Guthrie (1997) used a "shortened version” (p.423) of the measure. Because the relevant outcome for this study was behavioral engagement with reading, rather than print exposure regardless of student engagement, the RAI was framed in terms of self-selected reading. This approach provided information about the depth and breadth of reading students were doing outside of school or school requirements. Despite this modification, the fall and spring administrations of the RAI each had adequate reliabilities. The predictive validity of the RAI has been shown in relation both to reading achievement and intrinsic reading motivation (Cox \& Guthrie, 2001).

\section{Data Analysis}

\section{Principal Components Analysis}

I conducted a principal components analysis on the Motivations for Reading Questionnaire (MRQ) and Metacognitive Awareness of Reading Strategies Inventory (MARSI) to determine whether the subscales created by the measures' authors were represented in the data in the study. Each analysis yielded one factor, indicating that the survey items could appropriately be analyzed holistically, rather than broken down into separate scales. The format of the RAI does not allow for meaningful use of principal components analysis. For each of the three surveys, I used the student’s mean rating in subsequent analyses. 


\section{Analytic Approach: Hierarchical Linear Modeling}

Because students were nested within classrooms, I used Hierarchical Linear Modeling (HLM) to answer questions about how teacher- and student-level variables predicted student outcomes (i.e., Do students in classrooms where teachers incorporate more high conceptual press responses (or fewer reducing press responses) demonstrate stronger outcomes?). HLM is a better approach for analyzing nested data than is ordinary regression analysis because in estimating effects for a given classroom, it is designed to use take advantage of similar estimates available from other classrooms. Also, HLM allows for the partitioning of variation into within- and between-classroom components (Raudenbush \& Bryk, 2002, p.9). This partitioning facilitates the modeling of teacher-level effects (e.g., classroom discourse practices) on student outcomes (e.g., reading comprehension achievement, affective reading engagement).

I built HLM models to predict each of the four student outcome measures listed above. HLM models are built on multiple levels. In this study, Level-1 represented individual students and Level-2 represented classrooms or teachers. Level-1 (student) predictors included fall premeasure data, student gender, and student grade level. Level-2 predictors included teacher discourse patterns, school demographics, and class mean pretest scores and ratings. ${ }^{2}$

Level-2 estimates can be biased by Level-1 covariates that are related both to the outcome of the model and to some Level-2 predictor. Including the covariate in question, groupor grand-mean centered, at Level-1 and also including the mean score for a given Level-2 unit can eliminate this bias. For this reason, in building the model to predict Post-GMRT score, I entered students' Pre-GMRT score at Level-1 and the class mean Pre-GMRT score (GMRTPRE) at Level-2 to control for the possibility that other teacher-level predictors might be correlated with class mean Pre-GMRT scores. For example, it is possible that teachers with higher scoring 
students (on the Pre-GMRT) may use more high press discourse moves than teachers with lower scoring students. Because including the Level-2 class mean score serves to eliminate bias in estimates, I left it in the model regardless of whether it was a significant predictor of the outcome. For this reason, the class mean Pre-GMRT score was entered at Level-2 before model building continued at Level-1. I used this same procedure of entering the individual pre-measure score at Level-1 and the class mean for the same pre-measure at Level-2 before continuing with the analysis to build the models for all four student outcomes.

Once the individual and class mean pre-measure scores were entered into the model, the remaining Level-1 dummy variables (for gender (FEMALE) and grade level (GRADE4)) were entered in steps. Next, I entered the Level-2 demographic variables (percentage of students in the school identified as English Language Learners (ELLPRCNT), percentage of students in the school qualifying for free meals (FREEMEAL), proportion of students in the class who were fourth graders (PRP4TH) in steps along with the class mean pre-measure score.

At this point, I incorporated variables addressing the core hypotheses of the study into the model. I entered each of the four variables representing conceptual press (Expanded High Press Discourse, XHIGHPR; High Press Discourse, HIGHPR; Sustained High Press Discourse, SUSHIGHPR; and Reducing Press Discourse, REDPR) into the model independently. Because the existing research literature pointed to the positive contribution of strategy instruction to achievement and engagement and because the hypothesis of the study addressed conceptual press discourse, I also fit models in which each of the conceptual press variables was entered in combination with the strategy instruction variable (Strategy Instruction Discourse, SI), one pair at a time. The purpose was to investigate which version of the conceptual press variable in combination with the strategy instruction variable would best predict the post-measure score 
(accounting for students' individual pre-measure scores and class average pre-measure scores). At each stage of the analysis, non-significant effects were removed as necessary.

Results

\section{Descriptive Statistics}

The descriptive statistics for the teacher discourse patterns are displayed in Table 3. Each mean value represents the percentage of total utterances categorized as a given discourse pattern. For example, the mean percentage of total utterances that fell into the High Press Discourse pattern was $5.5 \%$. On average, about $14.7 \%$ of teacher utterances fell into the Reducing Press Discourse pattern. This relationship is displayed visually in Figure 1.

Note that the Expanded High Press category contains all the moves included in the High Press category, plus “Does that make sense?,” pinpointing, and other scaffolded follow-ups. As mentioned above, this category was included in the analysis because conceptual press discourse is a new construct and I wanted to examine two different ways of formulating it. High Press moves are a subset of Expanded High Press moves.

Similarly, Sustained High Press moves are a subset of High Press moves. The Sustained High Press category includes the same moves as the High Press category, but moves were only counted as Sustained High Press when they occurred over multiple turns with one child or small group. The purpose of including this variable in the analysis was to examine whether High Press moves might be stronger predictors of student outcomes when teachers used them in sustained interactions with individuals or small groups. The relationships among these three variables, then, is similar to that of nesting dolls, with Sustained High Press discourse acting as the smallest doll, High Press discourse as the medium sized doll, and Expanded High Press discourse as the largest doll. Only one of these variables was used at a time in the analysis. Unlike the High Press 
variables, Reducing Press and Strategy Instruction Discourse are mutually exclusive. Not all of the 29 discourse moves coded fell into any of these categories. Therefore, the mean percentages for the discourse patterns listed in Table 3 do not sum to $100 \%$.

Table 4 displays correlations among the teacher discourse patterns. Reducing Press Discourse was negatively correlated with all variations of High Press Discourse (High Press, Expanded High Press, and Sustained High Press, which were highly correlated with one another simply due to their nested relationship). The pattern of correlations suggests that most teachers could be categorized generally as either "high pressers" or as "press reducers.” An increased percentage of one of these patterns in a teacher's talk would naturally limit the other pattern, but there were a number of discourse moves that were coded as neither High Press nor Reducing press. A low percentage of High Press moves could have been associated with a high percentage of other moves, such as paraphrasing, evaluating student responses, or explaining how to use a comprehension strategy, rather than with a high percentage of Reducing Press moves.

Descriptive statistics for all dependent variables are displayed in Table 5. The mean PreGMRT score corresponds to a grade-level equivalent of approximately fifth grade, second month. The Post-GMRT score corresponds to a grade-level equivalent of approximately fifth grade, ninth month.

\section{Do Teacher Discourse Patterns Predict Student Outcomes?}

This study was designed to determine whether students in classrooms where teachers incorporate more high conceptual press responses (or fewer reducing press responses) demonstrate stronger outcomes in four areas: reading comprehension achievement (as measured by the GMRT); affective engagement (as measured by the MRQ); cognitive engagement (as measured by the MARSI); and behavioral engagement (as measured by the RAI). The following 
four sections report results for each of these four student outcome variables. See Table 6 for all four final models.

Reading Comprehension Achievement (GMRT). Reducing press discourse and strategy instruction discourse were both highly significant negative predictors of Post-GMRT score, according to the final model in Table 6. Students whose teachers used comparatively more reducing press discourse moves and comparatively more strategy instruction talk tended to demonstrate weaker comprehension achievement. The coefficient for reducing press discourse (-95.16) indicates that a student whose teacher used this discourse pattern for $100 \%$ of her utterances would be expected to score 95.16 points lower on the Post-GMRT than would a student whose teacher never used Reducing Press Discourse, holding constant all other variables in the model. In this study, the teacher who used Reducing Press moves most frequently did so for $25 \%$ of her total utterances. The teacher with the lowest Reducing Press frequency used these talk moves for $6 \%$ of her total utterances. The model predicts that a student whose teacher used Reducing Press Discourse for $25 \%$ of utterances would be expected to score 18.08 points (0.43 standard deviations) lower on the Post-GMRT than a student whose teacher used this discourse pattern for $6 \%$ of total utterances, controlling for other variables. Table 7 clarifies the relationship between Reducing Press Discourse and Post-GMRT score.

Similarly, the model predicts that a student whose teacher used Strategy Instruction Discourse for $100 \%$ of total utterances would score 62.64 points lower on the Post-GMRT than a peer whose teacher used no Strategy Instruction Discourse, all other relevant variables held constant. The actual range of Strategy Instruction Discourse found in this study was $4 \%$ to $34 \%$ of total utterances. The model predicts that a student whose teacher used the greatest proportion of Strategy Instruction Discourse in the study (34\%) would score 18.79 points (0.45 standard 
deviations) lower on the Post-GMRT than a student whose teacher used the smallest proportion of Strategy Instruction Discourse in the study (4\%).

When models were run with each of the High Press variables (Expanded High Press, High Press, and Sustained High Press) the coefficients all had positive magnitudes, but were not statistically significant. It is impossible to be certain why the High Press variables were not statistically significant predictors of Post-GMRT score, however, possible explanations are offered in the Discussion.

The final model for presented in Table 6 explains 99.91\% of the total between-classroom variance in Post-GMRT score. Of the variance that remained once the Pre-GMRT variables were incorporated into the model (3.08), this final model explains 92.86\%. Student grade and gender were not significant predictors of Post-GMRT score, nor were Level-2 demographic variables.

Affective Reading Engagement (MRQ). The final model for the Post-MRQ rating (presented in Table 6) includes student Pre-MRQ ratings, class mean Pre-MRQ ratings, and Reducing Press Discourse. Reducing Press Discourse is a marginally significant negative predictor of the Post-MRQ rating. This model predicts that a student whose teacher used the greatest proportion of Reducing Press Discourse in the study (25\% of the teacher's total utterances) would rate herself 0.15 points (0.33 standard deviations) lower on the Post-MRQ than a student whose teacher used the smallest proportion of Reducing Press Discourse in the study (6\% of the teacher's total utterances), holding the other variables in the model constant.

As was the case for the Post-GMRT model, coefficients for the three variables representing high conceptual press (Expanded High Press, High Press, and Sustained High Press) were positive but not statistically significant. None of the student- or teacher-level demographic variables was a statistically significant predictor of the Post-MRQ score. The final model 
explains 93.47\% of the total between-classroom variance in Post-MRQ rating. Of the variance that remained once the Pre-MRQ variables were incorporated into the model (0.0152), this final model explains $7.89 \%$.

Cognitive Reading Engagement (MARSI). The final model for the Post-MARSI rating includes student Pre-MARSI ratings, class mean Pre-MARSI ratings, and Reducing Press Discourse (see Table 6). Reducing Press Discourse is a marginally significant negative predictor of the Post-MARSI rating. The model predicts that a student whose teacher used the greatest proportion of Reducing Press Discourse in the study (25\% of the teacher's total utterances) would rate herself 0.27 points (0.57 standard deviations) lower on the Post-MARSI than a student whose teacher used the smallest proportion of Reducing Press Discourse in the study (6\% of the teacher's total utterances) holding the other variables in the model constant. Again, coefficients for the three variables representing high conceptual press (Expanded High Press, High Press, and Sustained High Press) were positive but not statistically significant.

The final model presented in Table 6 explains $61.65 \%$ of the total between-classroom variance in Post-MARSI rating. Of the variance that remained once the Pre-MARSI variables were incorporated into the model (0.0238), this final model explains $16.35 \%$.

Behavioral Reading Engagement (RAI). None of the teacher discourse variables were significant predictors of Post-RAI rating. The only significant predictors were Pre-RAI rating and the proportion of fourth graders in a student's class (as some classes included a mix of fourth and fifth grade students) (see Table 6). A greater proportion of fourth graders predicted higher Post-RAI ratings. Summary of Results 
The quantitative analyses and results presented indicate that Reducing Press Discourse was a significant negative predictor of three student outcomes: reading comprehension achievement, affective reading engagement, and cognitive reading engagement. While their coefficients were in the predicted positive direction in every case, none of the high conceptual press discourse variables (Expanded High Press, High Press, and Sustained High Press) was a statistically significant predictor of student outcomes. When entered into the model with Reducing Press Discourse, Strategy Instruction Discourse was a significant negative predictor of student Post-GMRT score. None of the teacher discourse variables predicted behavioral reading engagement.

Table 8 summarizes the findings of the HLM analyses with regard to conceptual press discourse. Each cell indicates the direction of the relationship between a given discourse pattern and a given outcome. It is worth noting that the sign in every cell is in the predicted direction.

\section{Discussion}

\section{High Press Discourse}

One of the most striking findings of this study was the sheer lack of high press discourse moves used by the 21 teachers. On average, only one out of twenty teacher utterances involved pressing students for clarification, elaboration, evidence, or examples. Teachers were nearly three times as likely to narrow their questions to make them easier, hint at the answer, answer their own questions, or let a student "off the hook" than they were to press students to think further with high press discourse moves. In some cases, only $2 \%$ of a teacher's utterances were categorized as high press moves.

High press discourse (in its three variable formats: High Press Discourse, Expanded High Press Discourse, and Sustained High Press Discourse) was expected to be associated with 
relatively greater improvements on all four student outcome measures. When these high press patterns were entered individually into the models for comprehension achievement and for all three forms of reading engagement, the coefficients carried the predicted positive signs. The nonsignificant results for the high conceptual press variables may simply indicate that the proportion of reducing press discourse a teacher uses is more important for improvement in comprehension achievement than is the proportion of high press discourse. However, three findings suggest that high press discourse merits further investigation as a predictor of student outcomes. First, Sustained High Press Discourse was a marginally significant $(p<.10)$ positive predictor of the Post-GMRT score (though the model incorporating Sustained High Press Discourse explained slightly less of the between-classroom variance than did the final model presented in Table 6).

Second, Reducing Press Discourse was strongly negatively and significantly correlated with each of the three variables representing high press discourse $(-0.55,-0.52,-0.73$, respectively). Teachers high in reducing press discourse tended to be low in high press discourse and vice versa. This relationship suggests that the negative relationship between reducing press discourse and comprehension achievement, affective engagement, and cognitive engagement implies a potential positive, if weaker, relationship between high press discourse and these student outcomes.

Finally, high press discourse may not have shown up as a statistically significant predictor of student outcomes in part due to the low frequency of high press discourse across all classrooms. Teachers in this naturalistic study rarely asked students for clarification, elaboration, evidence, or examples. Only two teachers used High Press Discourse for more than 9\% of utterances. One of these two teachers showed average class improvement on the GMRT of 18.81 points, nearly twice as many points as the average class improvement for all 21 classes $(9.78$ 
points). The low frequency and small range for High Press Discourse may have made it impossible to detect significant effects and indicates that further study of conceptual press discourse is warranted.

\section{Reducing Press Discourse, Achievement, and Engagement}

Reducing press discourse was associated with relatively low student outcomes on three of the four measures, holding constant prior student and class mean scores (and, in the case of comprehension achievement, the amount of strategy instruction provided by the teacher). More specifically, a greater proportion of reducing press talk was associated with weaker outcomes for comprehension achievement, affective reading engagement, and cognitive reading engagement. The discourse moves included in the Reducing Press Discourse pattern are: narrowing an initial question, offering hints, telling the answer, and calling on another student. The theory driving the categorization of these four discourse moves as reducing press was that given an initial question and student response, each of these moves serves to reduce the cognitive load on the student. Narrowing the initial question most often transforms an open-ended question into a multiple choice or either/or question, shrinking the universe of possible responses to two or three and changing the student's task from considering the initial question and articulating a response to selecting from a menu of options. Similarly, hints push the student in the direction of a desired or acceptable answer, taking away an opportunity for students to travel that cognitive distance on their own. Calling on another student (as when the first student offers an unacceptable or insufficient response) lets the first student "off the hook" and curtails the potential for pressing the student to think further or longer about the initial question and his own ideas. A student in a classroom where discourse around texts is characterized by frequent reduction in press may spend less time and expend less effort on thinking deeply and critically about texts. 


\section{Strategy Instruction Discourse and Comprehension Achievement}

The discourse moves included in the Strategy Instruction Discourse pattern were: providing explicit instruction about how to comprehend text or use comprehension strategies, identifying what students are doing strategically or metacognitively, and asking students to explain their use of strategies. Strategy instruction was associated with relatively poor reading comprehension achievement, holding constant prior student and class mean reading comprehension achievement and the proportion of reducing press discourse used by the teacher.

This result is particularly interesting in light of recent discussions in the field about the efficacy and quality of strategy instruction. While many studies have found that wellimplemented strategy instruction results in improved comprehension achievement as measured by standardized assessments including the GMRT (cf. Klingner, Vaughn, Arguelles, Hughes, \& Leftwich, 2004), it is unclear how this happens. It is possible that when students learn comprehension strategies, they read more actively and that this enhanced engagement, rather than their use of particular strategies, is the lever acting on their comprehension scores. Researchers have grown increasingly concerned about mechanistic strategy instruction that may in fact impede the larger enterprise of reading for meaning. Wilkinson and Son cite Garcia, Taylor, Pearson, Stahl and Bauer's (2007) finding that “teachers who were taught to implement strategy instruction during a year of professional development tended to 'get stuck,' overemphasizing strategies even as they were trying to foster students’ more responsive engagement with text” (2011, p. 366).

The negative relationship between strategy instruction and comprehension achievement in the present study may be explained in part by the approach used to measure strategy instruction. Most prior studies have contrasted a condition of strategy instruction with another 
approach to comprehension instruction. The focus has not generally been on teacher-student discourse, but on the broader instructional approach, with classrooms categorized either as using strategy instruction or not. In this study, strategy instruction varied continuously across classrooms and was based not on the overall approach but on actual observations of the frequency of particular utterance types. Also, because strategy instruction was not the focus of this study, there was no attention in the coding to the quality of strategy instruction delivered. Teacher utterances emphasizing surface-level use of strategies with an emphasis on correct use of tools like sticky notes were coded identically to teacher utterances emphasizing the use of strategies as tools for deep thinking about and interpretation of text. In both cases, coding simply reflected the frequency with which the teacher discussed comprehension strategies in any form. The findings reported here add to the complexity with which teachers and researchers are coming to understand strategy instruction. Further study is needed to help us understand how strategy instruction operates on reading comprehension and how it can best be implemented.

\section{Teacher Discourse and Behavioral Reading Engagement}

Contrary to the study hypothesis, none of the teacher discourse variables was a significant predictor of behavioral reading engagement. In this study, behavioral reading engagement was operationalized in terms of students' self-reported depth and breadth of reading free choice texts. The RAI was administered with instructions that students should report the reading they do that is not required for school or homework, but is just for fun or for their own interest. The purpose of framing the survey this way was to estimate how frequently and broadly students might read on their own without external requirements: their voluntary behavioral engagement with reading. Instructional discourse patterns did not appear to affect out-of-school, voluntary reading as measured by the RAI. It may be that a measure more proximal to classroom experiences, such as 
logs of time spent reading both in and out of school would have been more sensitive to variations in teacher-student discourse patterns.

\section{Limitations and Future Research}

This study has several limitations to note. First, no measure is perfect, and this is particularly true for tests of reading comprehension. The GMRT was selected to allow comparison with other studies, as it has been widely used in prior reading comprehension research, as well as for its strong validity and reliability. However, as a multiple-choice test, the GMRT is limited in its ability to provide information about how deeply or thoughtfully students are making sense of texts. Future studies might explore the effects of conceptual press discourse on student reading comprehension as measured by alternative assessments, such as written responses to reading or think-aloud protocols, allowing for more open-ended interpretation of texts. Such a measure might be more sensitive to the effects of teacher discourse patterns than was the GMRT.

All survey measures are limited by the accuracy of self-report and the effects of social desirability. Future studies might seek more immediate measures of engagement than those offered by surveys or reading logs. Videotapes of students during classroom instruction and stimulated recall interviews with students addressing their engagement would help researchers and educators to understand how conceptual press discourse may shape student affective and cognitive engagement “in the moment.” Future studies might build on Almasi, McKeown, and Beck’s (1996) approach of showing video of classroom events to students and asking them to identify and discuss instances when they or their peers appear engaged.

The observation protocol used in the study and the coding of teacher utterances during instruction certainly led to some errors in coding and occasional missed utterances. Had the 
resources been available, it would have been ideal to videotape every observation (rather than one observation per teacher) and conduct more complete discourse analysis on each tape in order to more confidently code each teacher utterance. However, the substantial pilot work and practice that went into the development of the protocol and the fact that protocol was used 63 times during the study adds credence to the claim that it captured patterns of teacher-student talk. The protocol seems more akin to a sketch of classroom talk than to a photograph. While a "photograph" would have been ideal, a sketch is a useful tool for understanding the shape and character of its subject. The observation protocol was imperfect but informative.

A key concern in selecting classrooms for the study was to ensure that it would be possible to observe reading comprehension instruction on a regular basis. While we might assume that reading comprehension would be taught in every fourth- and fifth-grade classroom, in fact the instruction (as opposed to the mere assessment) of reading comprehension is relatively rare (Durkin, 1978/1979; Pressley, 2006; Snow, 2002). As a result, the sample was skewed toward including classrooms in more affluent communities with smaller populations of English Learners. However, the total sample did include some classrooms in lower income communities with relatively high populations of English Learners. Overall, White, Asian American, and Latino students were well represented, while there were comparatively few African American children in the data set. Students in the sample displayed a wide range of incoming reading comprehension achievement on the Pre-GMRT, though the average score for the entire data set was relatively high.

\section{Implications}

For teachers interested in supporting their students' comprehension achievement and affective and cognitive reading engagement, the findings presented here act as a reminder that 
responses to their student's responses are an important facet of instruction. Of particular concern should be discourse practices that progressively reduce the cognitive load on students. When teachers respond to students by offering hints, narrowing questions to make them easier, providing the desired answer, or letting a student "off the hook" by simply calling on another student, students tend to show weaker outcomes. They report being less interested in reading, enjoying reading less, and using metacognitive reading strategies less frequently. They also demonstrate weaker reading comprehension on a standardized measure.

This study suggests that effecting positive achievement and engagement outcomes via shifts in classroom discourse may not require teachers to adopt multifaceted discourse-focused instructional programs. Even small changes in discourse, such as consciously limiting reducing press talk moves, may yield benefits for students. It appears from this study's findings that it may not be necessary to engage in lengthy interactions with individual children in order to garner the benefits of effective discourse. Apparently, asking even one follow-up question that does not reduce the cognitive load on students may support their achievement and engagement. This possibility should be encouraging to teachers in light of current constraints on instructional practices in literacy and the dominant role played by core reading programs in many school districts.

The results for strategy instruction present a more complicated picture for teachers. This study finds that increased use of strategy instruction predicts weaker reading comprehension achievement. In light of the consensus from experimental studies that quality instruction in multiple strategies supports achievement (cf., NICHD, 2000; Snow, 2002; Mason, 2004), this study's focus on classroom discourse raises questions not so much about the value of strategy instruction per se, as about the patterns of talk through which this instruction is enacted. There is 
a chance that the negative effects of strategy instruction found here are related more to the monologic nature of delivery than to the actual content. Teachers might experiment with more dialogic approaches to delivering strategy instruction, keeping in mind the value of doing some cognitive modeling and explicit instruction.

This initial exploration of conceptual press discourse opens up a variety of potentially productive avenues for future research. Given the limited proportion of high press discourse observed, research is needed to investigate whether and how teachers can incorporate high press discourse moves. For instance, an examination of the relationships between teacher discourse moves and student outcomes in classrooms where teachers use substantially larger proportions of high press discourse than did the teachers in this study would be worthwhile. An appropriate next step would be an intervention study in which teachers are trained to use high press discourse moves. Such an intervention study might contrast four conditions in which teachers are trained to use more high press discourse moves, trained to use reducing press moves less often, trained to do both, or not trained in classroom discourse.

Another important direction for future research would be qualitative examination of how high and reducing press discourse unfolds in classrooms and how teacher-student talk in the most effective classrooms (those with the greatest gains in comprehension achievement and reading engagement) compares turn-by-turn to teacher-student talk in less effective classrooms. Further, it will be useful to learn how teacher knowledge, beliefs, and epistemological orientations toward reading shape classroom discourse practices and how students respond emotionally and cognitively to different patterns of talk. Fine-grained discourse analysis of videotaped teacherstudent interactions will also be worthwhile in investigating the types of teacher talk moves associated with elaborated, complex student contributions. 
At its most fundamental level, like all instruction, comprehension instruction is composed of moment-to-moment interactions among students and between students and their teacher. The choices a teacher makes (consciously or unconsciously) about how to conduct classroom discourse around texts have potential consequences for students’ participation (Nassaji \& Wells, 2000), the degree to which they engage in thinking about texts (Nystrand, 1997), their understandings about what it means to be a reader (Johnston, Woodside-Jiron, \& Day, 2001), their identities as readers, their affective experiences of reading, and, ultimately, their proficiency as readers. As a construct, conceptual press discourse offers a useful lens through which to examine classroom talk and brings into focus the interplay among cognitive engagement, intrinsic motivation, and learning that is possible whenever teachers and students come together to talk about texts. The findings of this study, along with the powerful and complex nature of classroom discourse as a whole, call for further investigation of conceptual press discourse.

\section{References}

Almasi, J. F., McKeown, M. G., \& Beck, I. (1996). The nature of engaged reading in classroom discussions of literature. Journal of Literacy Research, 28(1), 107-146.

Baker, L., Dreher, M. J., \& Guthrie, J. T. (2000). Engaging Young Readers: Promoting achievement and motivation. New York: Guilford.

Baker, L. \& Wigfield, A. (1999). Dimensions of children's motivation for reading and their relations to reading activity and reading achievement. Reading Research Quarterly, 34(4), 452-477.

Beck, I.L. \& McKeown, M. G. (2006). Improving comprehension with Questioning the Author: A fresh and expanded view of a powerful approach. New York: Scholastic. 
Biskin, D. S., Hoskisson, K., \& Modlin, M. (1976). Prediction, reflection, and comprehension. The Elementary School Journal. 77, 131-139.

Blumenfeld, P. C., Puro, P., \& Mergendoller, J. R. (1992). Translating motivation into thoughtfulness. In H.H. Marshall (Ed.), Redefining student learning: Roots of educational change (pp.207-239). Norwood, NJ: Ablex.

Boyd, M. \& Rubin, D. (2006) How contingent questioning promotes extended student talk: The function of display questions. Journal of Literacy Research, 38(2), 141-169.

Cazden, C. (1988). Classroom discourse: The language of teaching and learning. Portsmouth, NH: Heinemann.

Chinn, C. A., Anderson, R. C., \& Waggoner, M. A. (2001). Patterns of discourse in two kinds of literature discussion. Reading Research Quarterly, 36(4), 378-411.

Connell, J. P., Spencer, M. B., \& Aber, J. L. Educational risk and resilience in African-American youth: Context, self, action, and outcomes in school. Child Development, 65, 493-506.

Cox, K. E. and Guthrie, J. T. (2001) Motivational and cognitive contributions to students’ amount of reading. Contemporary Educational Psychology, 26, 116-131.

Deci, E. L. \& Ryan, R. M. (1985). Intrinsic motivation and self-determination in human behavior. New York: Plenum Press.

Durkin, D. (1978/1979). What classroom observations reveal about reading comprehension instruction. Reading Research Quarterly, 15, 481-533.

Fiscal, demographic, and performance data on California’s K-12 schools. (2008). Retrieved December 23, 2008, from http://www.Ed-Data.k12.ca.us

Fredricks, J. A., Blumenfeld, P. C., \& Paris, A. H. (2004). School engagement: Potential of the concept, state of the evidence. Review of Educational Research, 74(1) 59-109. 
French, P. \& MacLure, M. (1981). Teachers’ questions, pupils’ answers: An investigation of questions and answers in the infant classroom. First Language, 2, 31-45.

Guthrie, J. T., McGough, K., \& Wigfield, A. (1994). Measuring reading activity: An inventory (Instructional Resource No. 4). Athens, GA: National Reading Research Center.

Guthrie, J. \& Wigfield, A. (2000). Engagement and motivation in reading. In M.L. Kamil. P.B. Rosenthal, P. D. Pearson, \& R. Barr (eds.), Handbook of reading research: Volume III (pp. 403-422). Mahwah, NJ: Erlbaum.

Guthrie, J. T., Wigfield, A., Metsala, J., \& Cox, K. (1999). Motivational and cognitive predictors of text comprehension and reading amount. Scientific Studies of Reading, 3(3), 231-256.

Johnston, P., Woodside-Jiron, H., \& Day, J. (2001). Teaching and learning literate epistemologies. Journal of Educational Psychology, 93(1), 223-233.

Kachur, R. \& Prendergrast, C. (1997). A closer look at authentic interaction: Profiles of teacherstudent talk in two classrooms. In M. Nystrand (Ed.), Opening dialogue: Understanding the dynamics of language and learning in the English classroom (pp. 75-88). New York: Teachers College Press.

Keene, E. O. \& Zimmerman, S. (1997). Mosaic of thought: Teaching comprehension in a reader's workshop. Portsmouth, NH: Heinemann.

Klingner, J. K., Vaughn, S., Arguelles, M. E., Hughes, M. T., \& Leftwich, S. A. (2004). Collaborative strategic reading: "Real-world” lessons from classroom teachers. Remedial and Special Education, 25, 291-302.

Lawrence, J. F. \& Snow, C. E. (2011). Oral discourse and reading. In M.L. Kamil. P.B. Rosenthal, P. D. Pearson, \& R. Barr (eds.), Handbook of reading research: Volume IV (pp. 320-338). New York: Routledge. 
MacGinitie, W., MacGinitie, R., Maria, R. K., Dreyer, L. G., \& Hughes, K. E. (2000). GatesMacGinitie reading tests (fourth edition). Itasca, IL: Riverside Publishing.

MacGinitie, W. H., MacGinitie, R. K., Maria, K., \& Dreyer, L. G. (2002) Gates-MacGinitie Reading Tests, Fourth Edition, Technical Report, Forms S and T. Itasca, IL: Riverside Publishing.

Mason, L. H. (2004). Explicit self-regulated strategy development versus reciprocal questioning: Effects on expository reading comprehension among struggling readers. Journal of Educational Psychology, 96, 283-296.

Mehan, H. (1979). Learning lessons. Cambridge, MA: Harvard University Press.

Mokhtari, K. \& Reichard, C. A. (2002). Assessing students’ metacognitive awareness of reading strategies. Journal of Educational Psychology, 94(2), 249-259.

Murphy, P. K., Wilkinson, I. A. G., Soter, A. O., Hennessey, M. N, \& Alexander, J. F. (2009). Examining the effects of classroom discussion on students' comprehension of text: A meta-analysis. Journal of Educational Psychology, 101(3), 740-764.

Nassaji, H. \& Wells, G. (2000). What's the use of “triadic dialogue”? An investigation of teacher-student interaction. Applied Linguistics, 21(3), 376-406.

National Institute of Child Health and Human Development. (2000). Report of the National Reading Panel. Teaching children to read: an evidence-based assessment of the scientific research literature on reading and its implications for reading instruction: Reports of the subgroups (NIH Publication No. 00-4754). Washington, DC: U.S. Government Printing Office.

Nystrand, M., \& Gamoran, A. (1991). Instructional discourse, student engagement, and literature achievement. Research in the Teaching of English, 25, 261-290. 
Nystrand, M. (1997). Opening dialogue: Understanding the dynamics of language and learning in the English classroom. New York: Teachers College Press.

Perencevich, K. (2004). The associations of autonomy support and conceptual press with engaged reading and conceptual learning from text. Unpublished doctoral dissertation, University of Maryland, College Park.

Pressley, M. (2006). Reading instruction that works: The case for balanced teaching. New York: Guilford Press.

Pressley, M., El-Dinary, P. B., Gaskins, I., Schuder, T., Bergman, J., Almasi, L., \& Brown, R. (1992). Beyond direct explanation: Transactional instruction of reading comprehension strategies. The Elementary School Journal, 92, (5), 513-555.

Raphael, T. E. \& McMahon, S. I. (1994). Book Club: An alternative framework for reading instruction. The Reading Teacher, 48, 102-116.

Raudenbush, S. W. \& Bryk, A. S. (2002). Hierarchical linear models: Applications and data analysis methods (2nd ed.). Newbury Park, CA: Sage Publications.

Rosenblatt, L. M. (1978). The reader, the text, the poem: The transactional theory of the literary work. Carbondale, Illinois: Southern Illinois University Press.

Skinner, E. A., Wellborn, J. G., \& Connell, J. P. (1990). What it takes to do well in school and whether I’ve got it: A process model of perceived control and children's engagement and achievement in school. Journal of Educational Psychology, 82, 22-32.

Snow, C. (2002). Reading for understanding: Toward an $R \&$ D program in reading comprehension. Arlington, VA: Rand Corporation.

Vygotsky, L. S. (1978). Mind in society: The development of higher mental psychological processes. Cambridge, MA: Harvard University Press. 
Wigfield, A., \& Guthrie, J. T. (1997). Relations of children's motivation for reading to the amount and breadth of their reading. Journal of Educational Psychology, 89, 420- 432.

Wilkinson, I. A. G. \& Son, E. H. (2011). A dialogic turn in research on learning and teaching to comprehend. In M.L. Kamil. P.B. Rosenthal, P. D. Pearson, \& R. Barr (eds.), Handbook of reading research: Volume IV (pp. 359-387). New York: Routledge.

\section{Footnotes}

1. Capitalized discourse patterns (e.g., High Press Discourse) refer to the variable as entered into HLM analysis. Non-capitalized patterns (e.g., high press discourse) refer to the general construct. 2. Ordinarily, school level data would constitute a third level in an HLM analysis. In this data set, very few schools had more than one participating classroom and no school had more than two participating classrooms, so school level variables were unique to the teacher in nearly every case. For this reason, school level variables were entered at Level-2 with other teacher variables. 